\title{
Submicroscopic Morphology of Protoplasm and its Derivatives*
}

\section{Cytoplasm}

Crot YTOPLASM is neither a true liquid nor a true solid, but has an interesting combination of characters which place it between the two states. It may contain as much as 97 per cent water, and in some circumstances exhibits streaming, or flow ; in contrast, it has some measure of rigidity, tensile strength, and elasticity. It is not perfectly elastic, however, but undergoes plastic deformation; the surface is sticky, and the material may be drawn into fine threads. To these properties must be added thixotropy (a change from the semi-solid to the semi-liquid state caused by stirring), imbibition, semipermeability, and optical anisotropy. Earlier attempts to explain the properties of cytoplasm, for example, the 'alveolar', 'granular', and 'fibrillar' hypotheses, have proved inadequate, and have been discarded in favour of a gel structure: whereas the former postulated structural units of microscopic dimensions, the latter involves a structure beyond microscopic resolution. A colloid is described as having a dispersed phase in a continuous dispersion medium. Ostwald describes nine possible combinations, since either of the two phases may be liquid, solid, or gaseous, and eight of these are recognized in Nature (that is, all except the gas-gas combination). A consideration of the properties of each fails to explain all the properties of cytoplasm, and it would appear that the substance cannot be an ordinary colloid with dispersed particles.

Within limits, the shape of the particles does not affect the properties of a dispersoid, but if they are filamentous, as indeed is known to be the case in cellulose and proteins, they will be in frequent contact with one another, and form a continuous framework, that is, a reticular system instead of a corpuscular one, so that both phases are continuous and mutually interpenetrating. It is essential that the 'framework phase' be solid, though the second phase may be either solid, liquid, or gaseous. In order to follow so far as possible the terminology of Nägeli, who laid the foundations on which the

- Prof. A. Frey-Wyssling, professor of botany in the Swiss Federal Institute of Technology, Zürich, delivered a series of three lectures at University College, London, on March 6, 7 and 9 . The contents of the flrst two, dealing respectively with the submicroscopic structure of oytoplasm and chloroplasts, are summarized in this article ; the principal contents of the third lecture, which treated the submicroscopic structure of cell walls, are covered by the article which appeared in NATURE, 142, 899-904 (1938); the lecture is to be published by Prof. Frey-Wyssling in the October (1939) number of Science Progres8. Details of the subject, with an extensive bibliography, are given in Prof. Frey-Wyssling's book, "Submikroskopische Morphologie des Protoplasmas und seiner Derivate" (Berlin : Gebrüder Borntraeger,
1938). present conceptions have been built, the framework phase is called the 'micellar framework', and the meshes of the network the 'intermicellar spaces'. The material occupying these spaces is called the 'imbibition medium', as distinct from the dispersion medium of ordinary colloids. Whereas reduction in size of the particles of a corpuscular dispersoid would, when molecular dimensions were reached, result in a true solution which would not have colloidal properties, a similar reduction in the dimensions of a micellar framework would simply mean a decrease in thickness of the micellar threads, leading ultimately to a molecular reticulum which would retain many of the properties of a colloid, and if the points of attachment were sufficiently numerous would still be a gel. It may now be considered how the known constituents of cytoplasm can be fitted into such a plan.

In addition to water and inorganic constituents, the cytoplasm consists of proteins, phosphatides, and lipoids. The fats, which may be taken as representative of the last group, are formed from fatty acids esterified with glycerol. The molecule may be pictured as a three-pronged fork (Fig. la), the prongs representing lipophilic, fatty acid chains. They have neither hydroxyl nor carboxyl groups, and, in consequence, are immiscible with water. The phosphatides, by way of contrast, are of a polar character. For example, lecithin is a glycerol ester with two fatty acid chains and one hydrophilic choline phosphate group. As the hydrophilic and lipophilic groups are mutually repellent, the molecule may be compared with a tuning fork having two lipophilic prongs and a hydrophilic handle (Fig. 1b). The proteins are polypeptide chains of indeterminate length with various side chains, which may be hydrophilic, lipophilic, acidic, or basic, according to the protein. These chains have three important characteristics :

(a) A repetition of similar links, eomparable with the metameric segmentation in zoology; (b) a great variety of composition due to differences in possible arrangement of the side chains; and (c) a reversible contractibility which is probably important in vital processes.

Of the three constituents mentioned, the protein chains alone have a structure capable of forming a molecular reticulum. Moreover, this framework is capable of carrying the other constituents of the cytoplasm in association with suitable side chains. Indications of possible 


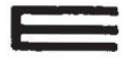

(a)

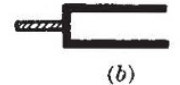

Fig. 1.

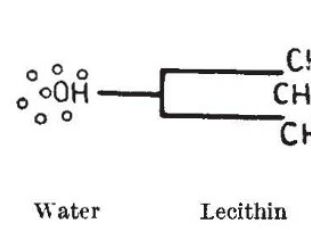

Lecithin
$\left.\mathrm{CH}_{3}\right\}$

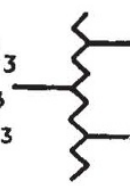

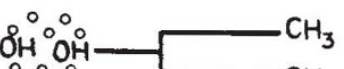<smiles></smiles>

Polypeptide chain Fig. 2.<smiles>CCCC</smiles>

Fat

combinations of this type are suggested in Fig. 2. Fig. 3 indicates types of union such as might occur at points of attachment of the chains themselves. Type I, between two lipophilic groups $\left(\mid-\mathrm{CH}_{3} \mathrm{H}_{3} \mathrm{C}-1\right)$ is a homopolar cohesion bond of the kind found in paraffin wax ; it would be affected by changes in temperature. Type II, between hydrophilic side chains $(|-\mathrm{OH} \ldots \mathrm{HO}-|)$ represents a heteropolar cohesion bond, or secondary valency bond; it may be loosened by heteropolar agents, and is capable of swelling. Type III, between acidic and basic side chains $\left(\left|-\mathrm{COO}^{-} \ldots \mathrm{NH}_{3}^{+}-\right|\right)$ forming a salt by ionic valency bonds, is affected by changes in hydrogen ion concentration. Type IV is a primary valency bond, and this particular type is dependent on redox reactions. A loose reticulum of the kind illustrated in Fig. 4, held by such bonds, would be sensitive to many kinds of change in environmental conditions.

The property of semipermeability may be controlled by lipoids incorporated in the protein network. These must be accumulated in the peripheral zone, because dyes which cannot penetrate the plasmatic surface spread freely in the cytoplasm when introduced by micro-injection. The high water content of cytoplasm together with mechanical rigidity, elasticity, and tendency to retain shape, are readily explained on the basis of a reticular framework, but would be difficult to account for on other systems. The properties of plasticity, viscosity, and protoplasmic streaming are less easily explained. It may be assumed

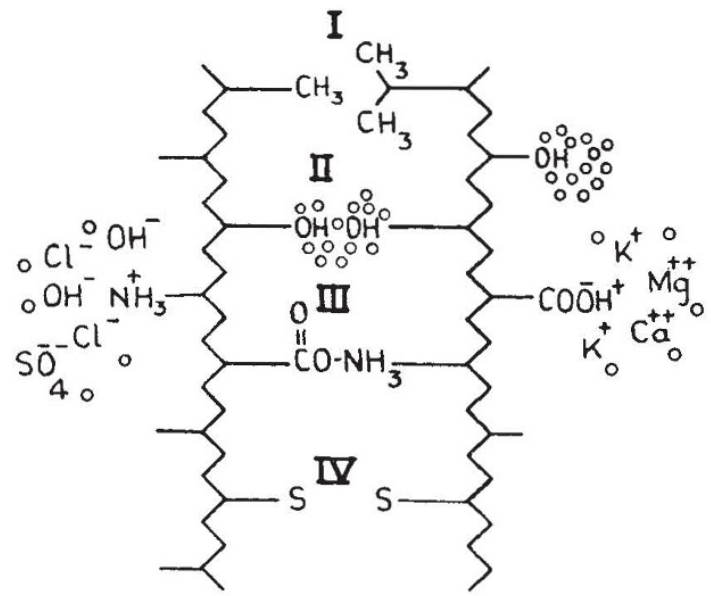

Fig. 3. that under certain conditions some of the bonds are loosened for a short time so that the micellar threads may slide over one another, and find new points of attachment. Not all the bonds would be loosened at the same time, for the polypeptide chains move in parallel groups as shown by the optical anisotropy, and this arrangement is responsible for the stickiness, tensile strength, and the possibility of being drawn into threads. Finally, Seifriz has shown that protoplasmic movement has a pulsating rhythm, and it is suggested that this is associated with the periodic contractions of the polypeptide chains.

\section{Chloroplasts}

Following the lines which appear to have been so successful in the investigation of cytoplasm, the molecular constitution of the main constituents of the chloroplasts was studied. Approximately half the chloroplast consists of protein, rather more than one third of lipoids, and about eight per cent of inorganic substances. The pigments (chlorophyll $a$ and $b$, xanthophyll and carotin) constitute only a very small proportion (for example, 1.5 per cent in tobacco leaves). It would appear probable that the proteins form a micellar reticulum as in the cytoplasm, and that the smaller molecules of the lipoids are lodged within the reticulum. The pigments are probably associated with the lipoids. The chlorophyll molecule may be likened to a signet, or rubber stamp, the disk representing a

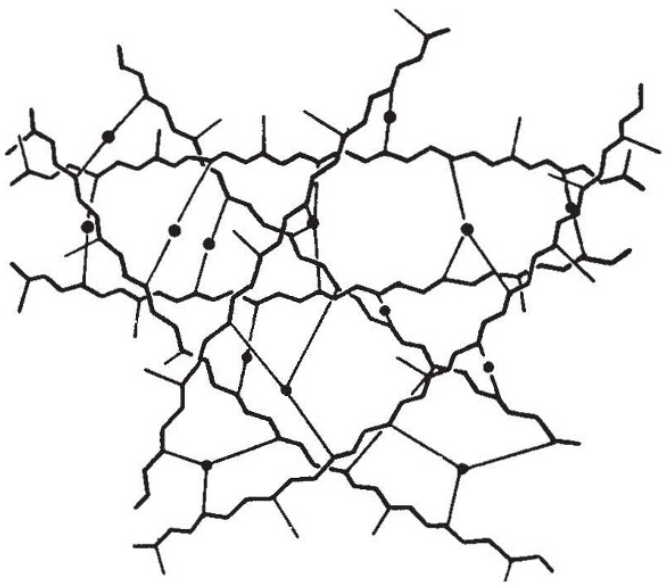

Fig. 4. 
so-called porphine ring, which is hydrophilic, and contains the nitrogen and magnesium of the chlorophyll, and the handle representing a lipophilic chain (Fig. 5a). The carotinoids are hydrocarbons $\left(\mathrm{C}_{40} \mathrm{H}_{56}\right)$, and are completely lipophilic. The xanthophylls are alcohols with one or more $\mathrm{OH}$ groups, and their affinity for water varies accordingly. Fig. $5 b$ is a diagrammatic representation of the molecule.

Some indications of the prob-

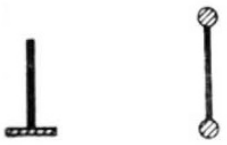

(a) Fig. 5 .

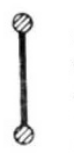

(b) able arrangement of these units within the chloroplast are afforded by the optical properties. From observation of double refraction, and of the changes of this property with change in the imbibition medium, it is inferred that the chloroplast is composed of submicroscopic layers, and that

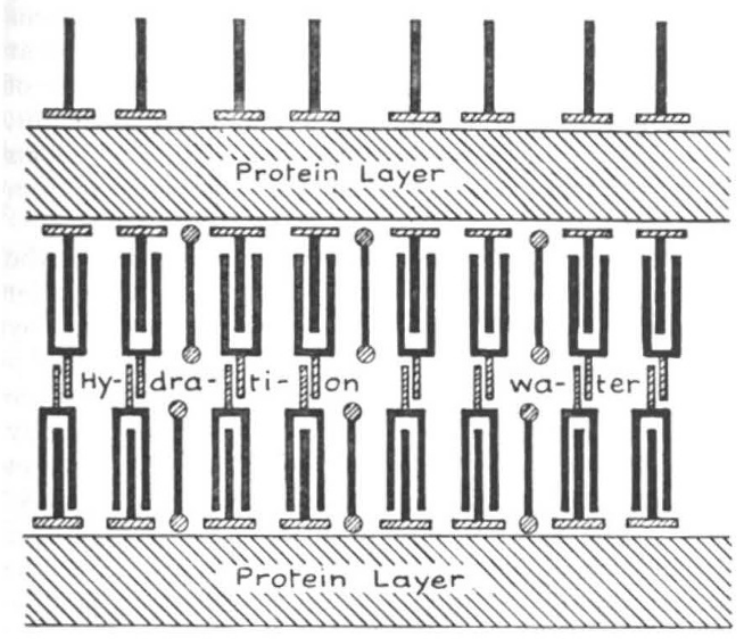

Fig. 6.

one type of layer consists of regularly orientated lipoid molecules. Since about half the chloroplast is protein, perhaps this alternates with the lipoid layers. Menke has actually observed a microscopic layering in some instances, and it would therefore appear likely that this suggestion is correct. Chlorophyll exhibits fluorescence when dissolved in alcohol or acetone, but the property is lost when water is added, because the molecules tend to form aggregates in which the lipophilic portions are directed towards one another and away from the water. Chlorophyll also exhibits fluorescence in the living chloroplast, and when in the form of mono-molecular layers on aluminium hydroxide, or with layers of lecithin. It is concluded that in the chloroplast chlorophyll occurs in layers, and, as already mentioned, in association with the lipoids.

Studies of the absorption of light by chlorophyll in the living chloroplast and various solvents, however, indicate that in the chloroplast it must occur in contact with an aqueous phase. Fig. 6 shows a possible arrangement of the units involved which satisfies all the requirements mentioned, including the association of the chlorophyll with the lipoids as required for fluorescence, and with an aqueous phase as required for light absorption. Moreover, the scheme is quite compatible with the findings of plant physiologists. Although some assert that the chlorophyll is concerned in the reduction of the carbon dioxide molecule, there is much evidence that its function is the capturing of energy from light. There is no stoichiometric relation between the carbon dioxide assimilated and the chlorophyll present, but for the assimilation of one molecule of carbon dioxide some 2,000 molecules of chlorophyll are involved. This number is called the assimilation unit, and it is estimated that even if the suggested layered system is complete within grana of about $0.5 \mu$ diameter, every chlorophyll layer might well contain about a hundred assimilation units.

Prof. Baur, of Zurich, has obtained traces of formaldehyde photolytically from carbon dioxide esterified with higher alcohols when brought together with a fluorescent pigment and a redox system. The method has striking parallels with the scheme outlined in Fig. 6. Molecular layers of chlorophyll mixed with cetyl alcohol in molecular proportions of about 1 : 30 were fixed by adsorption on threads of cellulose acetate. This system was dipped in a solution of methylene blue buffered with calcium carbonate, which acted as a redox medium (similar to the small amounts of ascorbic acid or iron compounds in the living chloroplast) in an atmosphere of carbon dioxide. In this system the chlorophyll appears simply to capture light, and may be replaced by other fluorescent dyes. It is important to notice also that the chemical reactions occur in a lipoid medium, as, for example, cetyl alcohol. Although Baur's system has an efficiency greatly removed from that of the chloroplast, it offers valuable contributions to fundamental knowledge.

\section{General Conclusions}

Evidence from chemistry, physics, and botany, indicates that many important constituents of plants are built of 'mixed systems'. The loose reticulum of the cytoplasm is as yet not well understood, but more is known of the cell wall with its 'rodlet mixed system', and the chloroplast with its layered, or 'platelet mixed system'. Such systems are not uncommon in other biological fields. Rodlet structure is found in the framework of chitin, silk fibroin, keratin, collagen tendons, 
muscle myosin, etc., and a stratified structure with alternating lipoid and protein layers in the sheaths of myelinated nerves, the rod-shaped cells of the retina, and the red blood corpuscles. It is interesting, moreover, to trace a 'linear principle' through chain-molecules, micelles, and fibrils, to fibres in the mechanical tissues, and a 'foliar principle' through molecular layers of pigments associated with layers of lipoids and proteins, in discoid grana in disk-shaped plastids which are exposed to light in a flattened leaf. The study of morphology must be pursued not simply to the somewhat arbitrary limit of microscopic resolution, but to the molecule, in which any change results in a change in the nature of the material itself.

\section{S. H. Clanke.}

\section{Obituary Notices}

\section{Mr. E. H. Richards}

$I^{T}$ is with deep regret that we record the death of Eric Hannaford Richards, of the Rothamsted Experimental Station, Herts, which occurred at Bovey Tracey on May 16.

Richards was born on June 28, 1878, at Hanwell, Middlesex, only son of Joseph Peek Richards, medical superintendent of the Middlesex County Asylum in 1867-92, and educated privately. In 1902 he joined the staff of the Royal Commission on Sewage Disposal, working under Dr. George McGowan, and was put in charge of the Commission's large-scale experiments at Dorking on different methods of sewage purification. These were brought to a successful conclusion. In 1909 he was transferred to Belfast to study methods of dealing with the excessive growth of seaweed in Belfast Lough : this was done in association with Prof. Letts of Queen's University. He then returned to Dr. McGowan's laboratory at Ealing, where he remained until 1913.

In that year, the Hon. Rupert Guinness (now Lord Iveagh) decided to arrange for investigations into the possibility of utilizing organic waste materials as fertilizers. His experience of the Bagshot sands at Woking had impressed upon him the need for making greater use of organic manure, while the growing tendency to substitute motors for horses was decreasing the supply of the stable manure which had hitherto been the mainstay of market gardeners, potato growers and dairy farmers around London. $\mathrm{He}$ saw, therefore, the need for opening up new supplies, and he provided the funds for the Rothamsted Experimental Station to carry out a full investigation. E.H. Richards was chosen for this work, and he fully justified the selection.

For the first few years, in collaboration with me, Richards studied the changes taking place in the making and storing of farmyard manure and showed how the losses occurred, and how also they might be reduced. Information was also obtained about the decomposition processes that occurred during the making and storing of the manure. This side of the work was greatly developed by an investigation made in association with $\mathrm{H}$. B. Hutchinson on the factors determining the rate and extent of the microbiological decomposition of straw. The essential conditions were an adequate supply of nutrients, particularly of nitrogen, which was generally deficient in the straw itself; not too acid a reaction ; and proper supplies of moisture and of air. The important practical result emerged that straw and other vegetable matter could be fairly rapidly decomposed with production of a humus manure by the simple expedient of adding a source of nitrogenous food for the micro-organisms bringing about the decomposition, and ensuring that the heap did not become too acid. The principles of compost-making were thus for the first time set out, and, although others have applied them in various ways, no new fundamental principles have been found.

One method of using the new principles, and the simplest that has yet been devised for use under British or similar conditions, was developed by Adco, an organization set up for this purpose by Lord Iveagh; it has been so successful that some 100,000 tons of manure are said to be made annually in this way, chiefly by private gardeners and market gardeners in Great Britain and by planters overseas. The method is also used in France for the making of mushroom beds. It is, however, less suitable for farmers, though modifications are from time to time devised which cheapen it.

In recent years Richards was also engaged on an investigation into the purification of effluents from sugar beet factories and milk factories. This was carried out for the Department of Scientific and Industrial Research in association with $\mathrm{D}$. Ward Cutler and his colleagues in the Microbiological Department at Rothamsted, and with S. H. Jenkins, who supervised the large-scale experiments, while Richards himself had as collaborator N. W. Barritt. This work led to the discovery of the conditions necessary for decomposition and purification of the effluents, and methods were then devised whereby these conditions could be secured in factory practice. The whole investigation affords an excellent example of successful collaboration of three groups of workers for the solution of a difficult technical problem.

All his life Richards had suffered from a deformity which might have crushed the spirit of a less courageous man. But his spirit was indomitable, and he let nothing stand in the way of the work he had set himself to do. He had perforce to renounce some of the pleasures of life, but he had the satisfaction of gaining in the highest degree the respect and affection of all who were associated with him. He was a loyal 